\title{
Numerical Method for Constructing Fixed Right Shift (FRS) Code for SAC-OCDMA Systems
}

\author{
Hassan Yousif Ahmed \\ Electrical Engineering Department \\ College of Engineering at Wadi Aldawaser, PSAU \\ Wadi Aldawasir, KSA
}

\author{
K. S. Nisar \\ Mathematics Department \\ College of Art and Science, PSAU \\ Wadi Aldawasir, KSA
}

\author{
Medin Zeghid \\ Electrical Engineering Department \\ College of Engineering at Wadi Aldawasir, PSAU \\ Wadi Aldawasir, KSA \\ S. A. Aljunid \\ School of Computer and Communication Engineering \\ Universiti Malaysia Perlis \\ Kangar, Malaysia
}

\begin{abstract}
In optical code division multiple access (OCDMA) systems, multiple access interference (MAI) problem which amplifies with the number of users actively involving in the network robustly bound the performance of such network. In this paper an algorithm to generate binary code sequences based on spectral amplitude coding (SAC) technique for CDMA under optical communication systems environment named fixed right shifting (FRS) is proposed. This algorithm is built with minimum cross correlation (MCC) using some type of Jordan matrices with straightforward algebraic methods. By using the code weight $W$ and the number of users $N$, various sets of binary code sequences for the possibilities of even and odd combination are constructed. Furthermore, this algorithm allows users with different code sequences to transmit data with minimum likelihood of interference. Simulation results show our technique for an agreeable bit error rate (BER) of $10^{-12}$ can support a higher number of users in deterministic and stochastic methods compared to reported techniques such Modified Quadratic Congruence (MQC) and Modified Frequency Hopping (MFH).
\end{abstract}

Keywords-FRS; OCDMA; MAI; BER; MFH; MQC

\section{INTRODUCTION}

An optical code division multiple access (OCDMA) is measured as a promising multi access technique to be implemented in high speed optical networks to enlarge the capacity of the optical communication system. OCDMA has many features such as simultaneous users, freedom of users involvement through allocation of distinct code sequence, bursty traffic management, and physical layer security because OCDMA system assigns each user a unique code [1-3]. OCDMA has many schemes appearing in literature reviews and among all of them incoherent systems have brought a lot of attention than coherent counterpart due to the handy ease of applying balance detection techniques [4-9]. In such system, each user is given a distinctive codeword based on the spectral amplitude as its code sequences. Once a user wishes to send " 1 ", it transmits out a codeword matching to the code sequence of the targeted receiver. At the recipient, all the codewords from involving users are matched. If correct codewords arrived, results with a high peak of autocorrelation are observed. On the other hand, cross correlation functions are taking place where multi access interference (MAI) is generated for incorrect codewords [1-3].

OCDMA system performance is analyzed by several quantitative parameters such as simultaneous users, stream rate, and magnitude of the powers to be injected in the transmitter and measured at the receiver and mode of transmission.

Researches on the SAC-OCDMA systems have led to the innovation of state of art codes with ideal cross correlation such as Modified Quadratic Congruence (MQC) and Modified Frequency Hopping (MFH). MQC code exists for a prime number while MFH code exists for a prime power. Furthermore, a restricted value of the weight limits the freedom of code construction [4-5].

Soma Kumawat et. al. proposed a general algorithm to construct both Modified Double Weight (MDW) code for even weights and Enhanced Double Weight (EDW) code for odd weights without mapping for any weight greater than 2 [7]. Nevertheless the number of users is reduced by half without mapping technique which means limited the capacity of the system employing such code for high number of users.

C. B. M. Rashidi et. al. [8] proposed Flexible Cross Correlation (FCC) code which proven to have better results compared to its counterparts in the literature reviews. FCC code has easier code structure, short code length for any number of users and weights. Nevertheless, the minimum cross-correlation value is two which degrades the system performance. Dynamic cyclic shift (DCS) code has been proposed in [6] with short code length to make cyclic operation. In order to accomplish the cyclic shift operation with a $\lambda c<=1$, the dynamic part $D$ should be greater than 7 else the $\lambda c$ value will exceed one. Limited codewords are generated as a result of the notion that the number of users equals to the code length.

Hilal Adnan Fadhil et al. [9] formed random diagonal (RD) code in easy construction steps with shortest code length by using code part and a data part. As the number of users increases, the value of $\lambda c$ exceeds one, ultimately impairs the 
system performance. On the other hand, as long as the value of $\lambda c$ between code sequences is big, phase-induced intensity noise (PIIN) arising from the square law photodetection of broadband source is too causing system performance deterioration [2-4]. In particular an MAI impact could be eliminated if code with a fixed value of $\lambda c$ is used by using subtraction technique $[2,3]$.

This paper introduces an efficient algorithm to construct FRS code family with $\lambda c<=1$. This algorithm allows users with different code sequences to transmit data with minimum likelihood of interference. FRS code is constructed based on modified Jordan block matrix with some algebraic techniques with a number of users $N$, code weights $W$, code length $L$ and cross correlation $\lambda \mathrm{c}$. The remaining parts of this paper are structured as follows. A mathematical model of the FRS code construction and its features are explained in Section 2. Section 3 shows the FRS's performance analysis. Calculated results are elaborated in Section 4. Study findings are drawn in Section 5.

\section{CODE CONSTRUCTION AND PROPERTIES}

In this section we first present the basic definitions, secondly the code design algorithm, then the steps of SFR code construction with the help of a Jordan Block (JB) and finally the code discussion with detailed examples.

\section{A. Definitions}

In linear Algebra, square matrix $\mathrm{A}$ is block diagonal if $\mathrm{A}$ has the form

$$
\left[\begin{array}{cccc}
A_{1} & 0 & \cdots & 0 \\
0 & A_{2} & \ddots & \vdots \\
\vdots & \ddots & \ddots & 0 \\
0 & \cdots & 0 & A_{k}
\end{array}\right\rfloor^{2}
$$

Where each $\mathrm{Ai}$ is a square matrix and the diagonals of each Ai lie on the diagonal of $\mathrm{A}$. Each $\mathrm{Ai}$ is called a block of $\mathrm{A}$. We modified this equation with the condition of (W-2) fixed right shifting of unity. Therefore, fixed right shift (FRS) code matrix is expressed in the light of A matrix as follows:

\section{FRS Matrix $=$}

$\left[\begin{array}{ccccccc}F N E_{1} & L N E_{1} & 0 & \cdots & \cdots & \cdots & 0 \\ 0 & F N E_{2} & L N E_{2} & 0 & \cdots & \cdots & 0 \\ 0 & 0 & F N E_{3} & \mathrm{LNE}_{3} & 0 & \cdots & 0 \\ \vdots & \vdots & 0 & \ddots & \ddots & \ddots & \vdots \\ \vdots & \vdots & \vdots & \ddots & \ddots & \ddots & 0 \\ 0 & 0 & 0 & \cdots & 0 & F N E_{N} & \mathrm{LNE}_{N}\end{array}\right]$

Where FNE represents first non-zero elements and LNE is the last non-zero element.

Step1: Form the arithmetic sequence (AS) as follows.

$$
A S=(W, W-1, W-2, W-3, W-4, \ldots, 1)
$$

\section{B. Strategy of FSR possibilities}

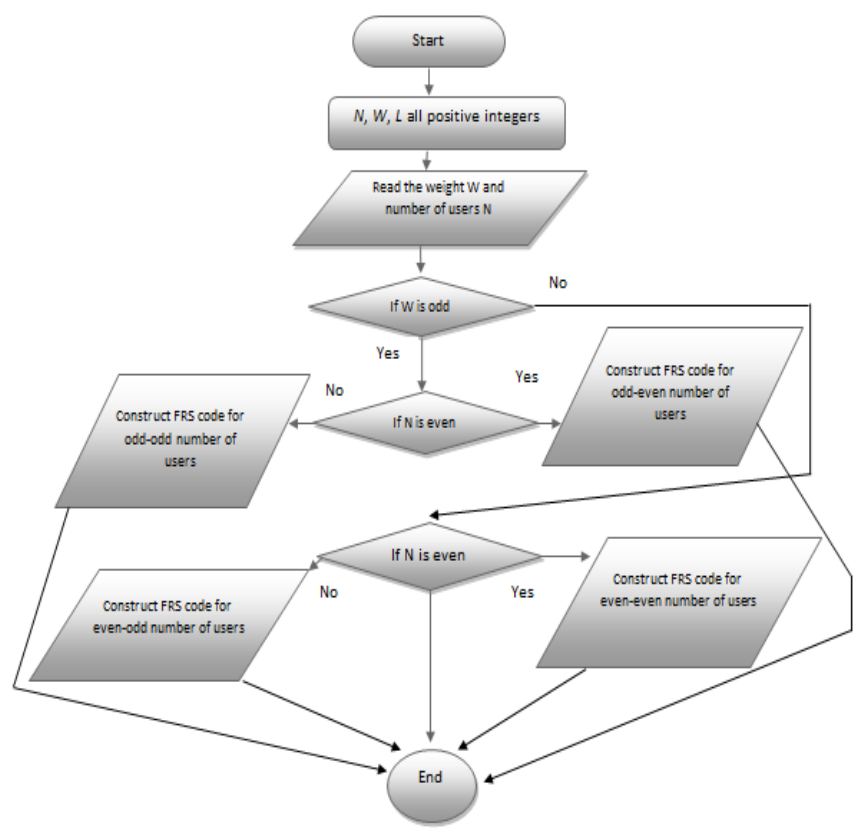

Fig. 1. Flowchart of FSR code construction

The flowchart below in Fig. 1 represents the major steps and points for FRS code family construction. The translation of this figure is given below in 11 steps.

1) Start

2) Declare integer variables, $(\mathrm{W}, \mathrm{N}, \mathrm{L})$

3) Get $\mathrm{W}$ and $\mathrm{N}$ values

4) If $\mathrm{W}$ is odd, go to step 5 else go to step 8.

5) If $\mathrm{N}$ is even, go to step 6 else go to step 7. END

6) Construct FRS code for odd-even case and then go to

7) Construct FRS code for odd-odd case and then go to END

8) If $\mathrm{N}$ is even, go to step 9 else go to step 10.

9) Construct FRS code for even-even case and then go to END

10)Construct FRS code for even-odd case

11)End

\section{Steps of FRS code construction:}

Step1: Calculate First non-zero elements (FNE) using

$$
(r, 1+(\mathrm{r}-1)(\mathrm{W}-2))
$$

Where $\mathrm{r}$ is the number of rows $(r=1,2,3, \ldots, N)$ and $W$ is the code weight.

Step 2: Calculate Last non-zero element (LNE) using

$(r, 1+(r-1)(W-2)+2 W-3)$

Step 3 : Fill ( $W-2)$ places with " 1 s" just after FNE and $(W$ 2) places with " $0 s$ " just before LNE.

Step 4 : Calculate the length using

$$
\mathrm{L}=N(W-2)+\mathrm{W}
$$


In order to explain the cross correlation properties, let us consider the examples in TABLES (I-IV) for the combination (even, even), (even, odd), (odd, odd), (odd, even).

D. Code Examples

Example 1: Odd, Odd $(W=3, N=7)$

TABLE I. FSR CODE PATTERNS FOR ODD-ODD CASE

\begin{tabular}{|l|l|l|l|l|}
\hline $\begin{array}{l}\text { No.of } \\
\text { rows }\end{array}$ & $\begin{array}{l}\text { FNE } \\
\text { Lower } \\
\text { bounds }\end{array}$ & $\begin{array}{l}\text { LNE } \\
\text { Upper } \\
\text { bounds }\end{array}$ & $\begin{array}{l}\text { Code } \\
\text { Position }\end{array}$ & Code \\
\hline 1 & 1 & 4 & $12 \times 4$ & 1101000000 \\
\hline 2 & 2 & 5 & $23 \times 5$ & 0110100000 \\
\hline 3 & 3 & 6 & $34 \times 6$ & 0011010000 \\
\hline 4 & 4 & 7 & $45 \times 7$ & 0001101000 \\
\hline 5 & 5 & 8 & $56 \times 8$ & 0000110100 \\
\hline 6 & 6 & 9 & $67 \times 9$ & 0000011010 \\
\hline 7 & 7 & 10 & $78 \times 10$ & 0000001101 \\
\hline
\end{tabular}

Example 2: Even, Odd. ( $W=3, N=7)$

TABLE II. FSR CODE PATTERNS FOR EVEN-ODD CASE

\begin{tabular}{|l|l|l|l|l|}
\hline $\begin{array}{l}\text { No.of } \\
\text { rows }\end{array}$ & $\begin{array}{l}\text { FNE } \\
\text { Lower } \\
\text { bounds }\end{array}$ & $\begin{array}{l}\text { LNE } \\
\text { Upper } \\
\text { bounds }\end{array}$ & $\begin{array}{l}\text { Code } \\
\text { Position }\end{array}$ & Code \\
\hline 1 & 1 & 6 & $123 \times x 6$ & 111001000000000000 \\
\hline 2 & 3 & 8 & $345 \times x 8$ & 001110010000000000 \\
\hline 3 & 5 & 10 & $567 \times x 10$ & 000011100100000000 \\
\hline 4 & 7 & 12 & $789 \times x 12$ & 000000111001000000 \\
\hline 5 & 9 & 14 & $91011 \times x 14$ & 000000001110010000 \\
\hline 6 & 11 & 16 & $111213 \times x 16$ & 000000000011100100 \\
\hline 7 & 13 & 18 & $131415 \times 18$ & 000000000000111001 \\
\hline
\end{tabular}

Example 3: Odd, Even. ( $W=3, N=7)$

TABLE III. FSR CODE PATTERNS FOR ODD-EVEN CASE

\begin{tabular}{|l|l|l|l|l|}
\hline $\begin{array}{l}\text { No.of } \\
\text { rows }\end{array}$ & $\begin{array}{l}\text { FNE } \\
\text { Lower } \\
\text { bounds }\end{array}$ & $\begin{array}{l}\text { LNE } \\
\text { Upper } \\
\text { bounds }\end{array}$ & $\begin{array}{l}\text { Code } \\
\text { Position }\end{array}$ & Code \\
\hline 1 & 1 & 8 & $1234 \times x \times 8$ & 11110001000000000000000000 \\
\hline 2 & 4 & 11 & $4567 \times x x 11$ & 00011110001000000000000000 \\
\hline 3 & 7 & 14 & $78910 \times x \times 14$ & 00000011110001000000000000 \\
\hline 4 & 10 & 17 & $10111213 \times x \times 17$ & 00000000011110001000000000 \\
\hline 5 & 13 & 20 & $13141516 \times x \times 20$ & 00000000000011110001000000 \\
\hline 6 & 16 & 23 & $16171819 \times x \times 23$ & 00000000000000011110001000 \\
\hline
\end{tabular}

Example 4: Even, Even $(W=4, N=6)$
TABLE IV. FSR CODE PATTERNS FOR EVEN-ODD CASE

\begin{tabular}{|l|l|l|l|l|}
\hline $\begin{array}{l}\text { No.of } \\
\text { rows }\end{array}$ & $\begin{array}{l}\text { FNE } \\
\text { Lower } \\
\text { bounds }\end{array}$ & $\begin{array}{l}\text { LNE } \\
\text { Upper } \\
\text { bounds }\end{array}$ & $\begin{array}{l}\text { Code } \\
\text { Position }\end{array}$ & Code \\
\hline 1 & 1 & 6 & $123 \times x 6$ & 111001000000000000 \\
\hline 2 & 3 & 8 & $345 \times x 8$ & 001110010000000000 \\
\hline 3 & 5 & 10 & $567 \times x 10$ & 000011100100000000 \\
\hline 4 & 7 & 12 & $789 \times x 12$ & 000000111001000000 \\
\hline 5 & 9 & 14 & $91011 \times x 14$ & 000000001110010000 \\
\hline 6 & 11 & 16 & $111213 \times x 16$ & 000000000011100100 \\
\hline
\end{tabular}

Let $x$ represents desired user, based on above tables the following properties are depicted as follows:

Property 1:

The cross correlation is one between the first three users.

Property 2:

For $x=2$ the cross correlation is one between \#userx and \#userx-1, \#userx+1, and \#userx+2.

Property 3:

For $x \geq 3$ the cross correlation is one between \#userx, \#userx-1, \#userx-2, \#userx+1, and \#userx+2.

Property 4:

This Code satisfies the condition of $(W-2)$ fixed right shift of unity. Here the minimum code weight is 3 and we developed the code according to $(W-2)$ fixed right shift of unity.

\section{PERFORMANCE ANALYSIS OF THE FSR CODE SySTEM}

The transmitter/receiver structure based on the FSR code sequence for $W=3$ is shown Fig. 2. As listed in TABLE I, the information of user\#1 which was coded as 110100000 has been modulated using ON-OFF Keying (OOK) technique as shown in Fig. 2. The optical pulses are then reflected to an FBG set, where specific wavelengths $\left(\lambda_{1} \lambda_{2} \lambda_{4}\right)$ are assigned to the chips of specific code given to the desired user. In the code sequences the positions of the " $1 \mathrm{~s}$ " determine the center wavelengths of FBGs. In Fig. 2 the received optical pulses are decoded by the corresponding decoder. For the data to be recovered the decoder should have the same spectral response 
to the intended encoder [3]. The detected sequences comprise the FSR code spectrum of the desired user in company with overlapping spectra from other interference of FSR code sequences. The complementary wavelengths, $\lambda_{3} \lambda_{5} \lambda_{6} \lambda_{7} \lambda_{8} \lambda_{9}$ of the intended user are detected by the complementary decoder where the received wavelengths are processed via
FBG sets. Subsequently the results are circulated to balanced photo-detectors [3]. A subtraction process is needed where a subtractor is used to strike the unwanted from the wanted signal. Finally, the original data is recovered after photo detections, low pass filter (LPF) and thresholding processes.

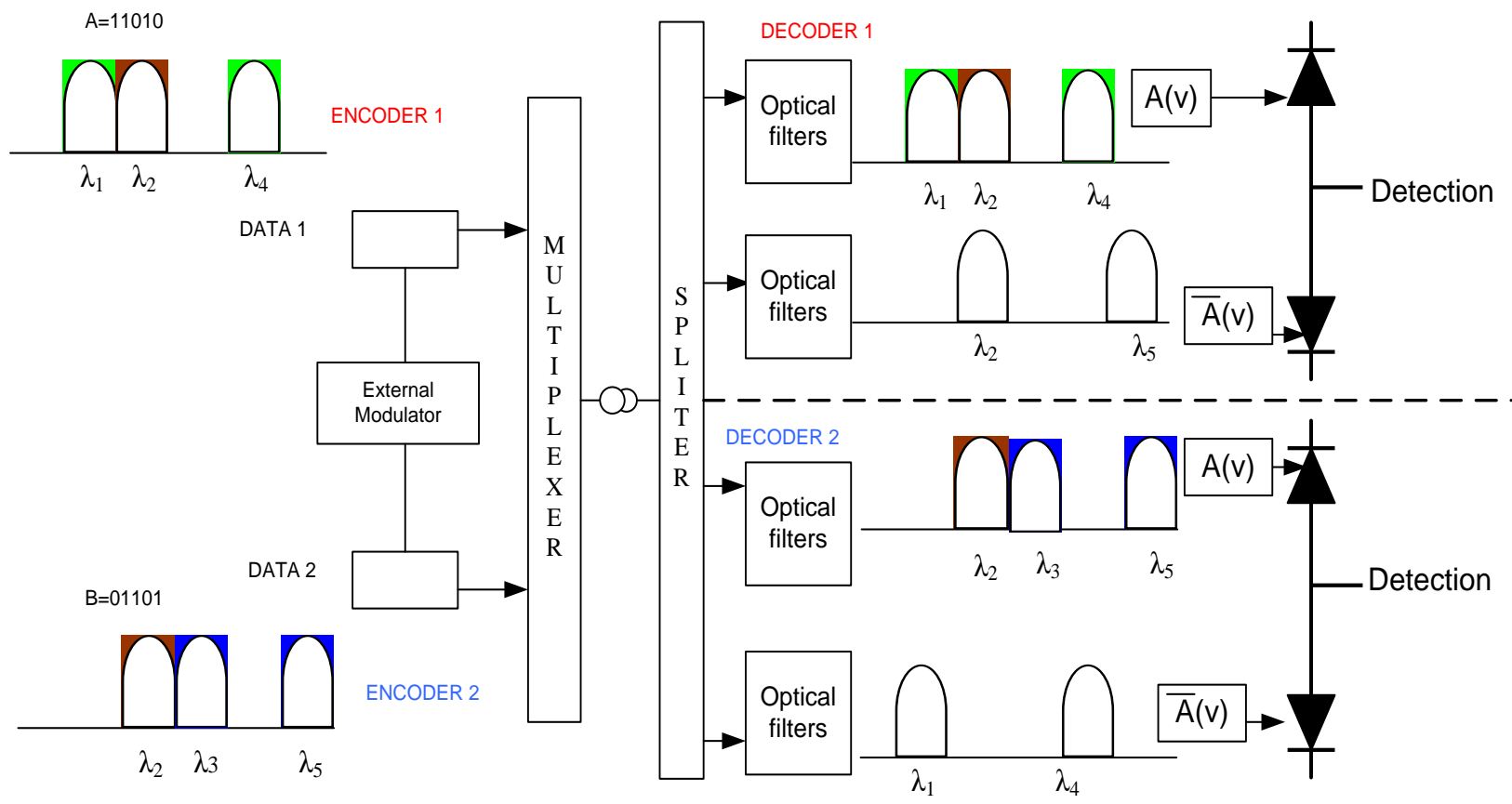

Fig. 2. Execution of the XOR detection technique using FSR code, transmitter- receiver

If $\mathrm{Cf}(\mathrm{i})$ indicates the ith element of the fth SFR code word, based on XOR subtraction the code properties are given as [910]:

$$
\sum_{i=1}^{L} C_{f}(i) C_{g}(i)= \begin{cases}W, & f=g \\ 1, & f \neq g \\ 0, & f \neq g\end{cases}
$$

$$
\sum_{i=1}^{L}\left(C_{f}(i) \oplus C_{g}(i)\right) \bullet C_{f(i)}= \begin{cases}0, & f=g \\ W-1, & f \neq g \\ 0, & f \neq g\end{cases}
$$

The condition of $f=g$ meaning the addition operation of FNE and LNE $(\mathrm{FNE}+\mathrm{LNE}=W$ ) for targeted user whereas the condition $f \neq g$ has two choices. The first choice is the interference users at following FNEs of targeted user when $\lambda \mathrm{c}$ $=1$. The second choice is the non-interference users for $\lambda \mathrm{c}=0$. Thus, the XOR procedure of $(\mathrm{Cf}(\mathrm{i}) \oplus \mathrm{Cg}(\mathrm{i}) \bullet \mathrm{Cf}(\mathrm{i}))$ is satisfied for $\mathrm{f} \neq \mathrm{g}$ only. However, the $\lambda \mathrm{c}$ of $(\mathrm{Cf}$ (i) $\oplus \mathrm{Cg}$ (i) $\bullet \mathrm{Cf}(\mathrm{i}))$ is satisfied for $\mathrm{f} \neq \mathrm{g}$ only in Eq. (8) while from Eq. (7), the cross correlation of $\mathrm{Cf}$ (i). $\mathrm{Cg}$ (i) is $\mathrm{W}$ when $\mathrm{f}=\mathrm{g}$. Consequently, the MAI can be removed as the cross correlation

$$
\begin{aligned}
& \left.\sum_{i=1}^{L}\left(C_{f}(i) \oplus C_{g}(i)\right) \bullet C_{f}(\mathrm{i})\right) \quad \text { could be subtracted from } \\
& \sum_{i=1}^{L} C_{f}(i) C_{g}(i) \quad \text { when } \mathrm{f} \neq \mathrm{g} \text {. Hence, the original information is }
\end{aligned}
$$

recovered for any decoder that computes Eq. (9) to decline the MAI impact.

Thus

$$
\sum_{i=1}^{L} C_{f}(i) C_{g}(i)-\frac{\sum_{i=1}^{L}\left(C_{f}(i) \oplus C_{g}(i)\right) \bullet C_{f(i)}}{W-1}= \begin{cases}W, & f=g \\ 0, & f \neq g\end{cases}
$$

Hence, the weight is zero when $f \neq g$, meaning MAI can be fully removed by using the XOR subtraction detection technique.

Thus, the result is zero when $f \neq g$ which means MAI impact is fully eliminated by using the XOR scheme.

Once a wide pulse is directed to FBGs group, the 
incoherent light fields are mixed and incident at a photodetector, the phase noise of the fields makes an intensity noise at the output of photo-detector [4-6]. The coherence time of a thermal source $(\tau \mathrm{c})$ is given by [11]:

$$
\tau_{c}=\frac{\int_{0}^{\infty} G^{2}(v) d v}{\left.\left\lceil\int_{0}^{\infty} G(v) d v\right\rfloor\right\rceil^{2}}
$$

where $^{G(v)}$ is the single sideband power spectral density (PSD) of the source. The variance of photocurrent as a cause of an unpolarized thermal light detection is given as:

$$
\left\langle i^{2}\right\rangle=\left\langle I_{\text {shot }}^{2}\right\rangle+\left\langle I_{\text {PIIN }}^{2}\right\rangle+\left\langle I_{\text {thermal }}^{2}\right\rangle
$$

Where $I_{\text {shot }}^{2}$ indicates shot noise, $I_{P I I N}^{2}$ is phase induced intensity noise and $I_{\text {thermal }}^{2}$ is the thermal noise. Thus, Eq. (12) is given as:

$$
\left\langle i^{2}\right\rangle=2 e I B+I^{2} B \tau_{c}+4 K_{B} T_{n} B R_{L}
$$

Where electron charge $e$; average photocurrent I; coherence time of the source $\tau_{\mathrm{c}}$; noise-equivalent electrical bandwidth of the receiver $B$; Boltzmann's constant $K B$; absolute receiver $T_{\mathrm{n}}$ noise temperature; receiver load resistor $R_{\mathrm{L}}$. The power spectral density (PSD) of the received optical signals can be written as [5-6]:

$$
r(v)=\frac{P_{s r}}{\Delta v} \sum_{n=1}^{N} d_{n} \sum_{i=1}^{L} c_{n}(i) \operatorname{rec}(i)
$$

where $P_{\mathrm{sr}}$ is the effective power of a broad-band source at the receiver, $N$ is the number of users, ${ }^{d}{ }_{n}$ is the data bit of the $n$th user that is " 1 " or " 0 ", and $L$ is the FRS code length. The rec ( $i$ ) function in Eq. (14) is given by

$$
\operatorname{rec}(i)=u\left[v-v_{o}-\frac{\Delta v}{2 L}(-L+2 i-2)\right]-u\left[v-v_{o}-\frac{\Delta v}{2 L}(-L+2 i)\right]
$$

From Eq. (15), $u[v]$ is the unit step function and given as:

$$
u[v]= \begin{cases}1, & v \geq 0 \\ 0, & v<0\end{cases}
$$

The total power incident at the input of $P D_{1}$ and $P D_{2}$ of Fig. 2 of the $g$ th receiver through 1 bit interval is given by

$$
\begin{aligned}
& \left.\int_{0}^{\infty} G_{1}(v) d v=\int_{0}^{\infty} \frac{P_{s r}}{\Delta V} \sum_{f=1}^{N} d_{f} \sum_{i=1}^{L} c_{f}(i) c_{g}(i) \mid \begin{array}{l}
\mid u\left[v-v_{o}-\frac{\Delta v}{2 L}(-L+2 i-2)\right] \\
-u\left[v-v_{o}-\frac{\Delta v}{2 L}(-L+2 i)\right]
\end{array}\right\} d v \\
& =\frac{P_{s r}}{\Delta V} \frac{\Delta v}{L} \sum_{f=1}^{N} d_{f} \sum_{i=1}^{L} c_{f}(i) c_{g}(i) \\
& =\frac{P_{s r} W}{L} d_{g}+\frac{P_{s r}}{L} \sum_{f=1, f \neq g}^{N} d_{f} \\
& \left.\int_{0}^{\infty} G_{2}(v) d v=\int_{0}^{\infty} \frac{P_{s r}}{\Delta V} \sum_{f=1}^{N} d_{f} \sum_{i=1}^{L} \frac{c_{f}(i)\left(c_{f}(i) \oplus c_{g}(i)\right)}{W-1} \mid \begin{array}{l}
\mid u\left[v-v_{o}-\frac{\Delta v}{2 L}(-L+2 i-2)\right\rceil \\
-u\left\lfloor v-v_{o}-\frac{\Delta v}{2 L}(-L+2 i)\right\rfloor
\end{array}\right\} d v \\
& =\frac{P_{s r}}{\Delta V} \frac{\Delta v}{L} \sum_{f=1}^{N} d_{f} \sum_{i=1}^{L} \frac{c_{f}(i)\left(c_{f}(i) \oplus c_{g}(i)\right)}{W-1} \\
& =\frac{P_{s r}}{L} \sum_{f=1, f \neq g}^{N} d_{f}
\end{aligned}
$$

The current I of the difference of photodiodes for desired user is calculated as

$$
I=I_{1}-I_{2}
$$

where $^{I_{1}, I_{2}}$ are the currents at $P D_{1}$ and $P D_{2}$, respectively.

$$
\begin{aligned}
I & =\Re \int_{0}^{\infty} G_{1}(v) d v-\Re \int_{0}^{\infty} G_{2}(v) d v \\
& =\Re\left(\frac{P_{s r} W}{L} d_{g}+\frac{P_{s r}}{L} \sum_{f=1, f \neq g}^{N} d_{f}-\frac{P_{s r}}{L} \sum_{f=1, f \neq g}^{N} d_{f}\right) \\
& =\Re\left(\frac{P_{s r} W}{L} d_{g}\right)
\end{aligned}
$$

where $\mathfrak{R}$ is the responsivity of the photodetectors given by

$$
\mathfrak{R}=\frac{\eta e}{h V_{c}}
$$

The quantum efficiency $\eta$, the electron charge $e$, the Planck's constant $h$, and the central frequency of the original broad-band optical pulse $V \mathrm{c}$. The shot noise power is given as: 


$$
\begin{aligned}
\left\langle I_{\text {shot }}^{2}\right\rangle & \left.=2 e B \Re\left|\int_{0}^{\infty} G_{1}(v) d v+\int_{0}^{\infty} G_{2}(v) d v\right|\right] \\
& =2 e B \Re\left(+\frac{P_{s r}}{L} \sum_{f=1, f \neq g}^{N} d_{f}+\frac{P_{s r}}{L} \sum_{f=1, f \neq g}^{N} d_{f}\right) \\
& \left.=2 e B \Re \frac{P_{s r}}{L} \mid W d_{g}+2 \sum_{f=1, f \neq g}^{N} d_{f}\right) \\
& =2 e B \Re \frac{P_{s r}}{L}(W+2(N-1)) \\
& \left\langle I_{\text {shot }}^{2}\right\rangle=2 e B \Re \frac{P_{s r}}{L}[2(N-1)+W]
\end{aligned}
$$

Applying the same process given in [2-3] and simplifying the summation from Eq. (12), once all the users are sending "'1", using the average value as $\sum_{f=1}^{N} C_{f} \cong \frac{N W}{L}$ and therefore the PIIN noise power is given as:

$$
\begin{aligned}
& \left\langle I_{\text {PIIN }}^{2}\right\rangle=B I_{1}^{2} \tau_{c 1}+B I_{2}^{2} \tau_{c 2} \\
& =B \Re^{2}\left[\int_{0}^{\infty} G_{1}^{2}(v) d v+\int_{0}^{\infty} G_{2}^{2}(v) d v\right] \\
& =B \Re^{2} \frac{P_{s r}^{2}}{\Delta v L} \sum_{i=1}^{L}\left\{C_{g}(i)\left\lceil\sum_{f=1}^{N} d_{f} C_{f}(i)\right\rceil \cdot\left\lceil\sum_{m=1}^{N} d_{m} C_{m}(i)\right\rceil\right\} \\
& +\frac{B \Re^{2}}{L} \frac{P_{s r}^{2}}{\Delta v L} \sum_{i=1}^{L}\left\{\left(C_{f}(i) \oplus C_{g}(i)\right)\left[\sum_{f=1}^{N} d_{f} C_{f}(i)\right\rceil\left\lceil\sum_{m=1}^{N} d_{m} C_{m}(i)\right]\right\} \\
& \left.\cong B \Re^{2} \frac{P_{s r}^{2}}{\Delta v L} \sum_{i=1}^{L}\left\{C_{g}(i) \frac{N W}{L} \mid \sum_{f=1}^{N} C_{f}(i)\right)\right\} \\
& +\frac{B \Re^{2}}{L} \frac{P_{s r}^{2}}{\Delta v L} \sum_{i=1}^{L}\left\{\left(C_{f}(i) \oplus C_{g}(i)\right) \frac{N W}{L}\left(\sum_{f=1}^{N} C_{f}(i)\right)\right\} \\
& \cong B \Re^{2} \frac{P_{s r}^{2}}{\Delta v L} \frac{N W}{L} \sum_{f=1}^{N}\left(\sum_{i=1}^{L} C_{f}(i) \cdot C_{g}(i)\right) \\
& +\frac{B \Re^{2}}{\Delta v L} \frac{P_{s r}^{2}}{L} \frac{N W}{f} \sum_{f=1}^{N}\left(\sum_{i=1}^{L} C_{f}(i) \cdot\left(C_{f}(i) \oplus C_{g}(i)\right)\right) \\
& \cong \mathrm{B}^{2} \frac{P^{2}{ }_{s r}}{\Delta V L} \frac{N W}{L} \sum_{f=1}^{N}\left(\sum_{i=1}^{L} C_{f}(i) C_{g}(i)\right)_{+\mathrm{B}} \mathfrak{R}^{2} \frac{P^{2}{ }_{s r}}{\Delta V L} \frac{N W}{L} \\
& \sum_{f=1}^{N}\left\lceil\sum_{i=1}^{L} C_{f}(i)\left(C_{f}(i) \oplus C_{g}(i)\right)\right\rceil \\
& =\mathrm{B} \mathfrak{R}^{2} \frac{P^{2}{ }_{s r}}{\Delta V L} \frac{N W}{L}[W+1+(N-1)+(\mathrm{N}-1)]
\end{aligned}
$$

The maximum MAI for preferred user will be $N-4$. As a result, Eq. (23) can be rewritten as:

$$
\left\langle I_{P I I N}^{2}\right\rangle=\Re^{2} \quad \frac{P^{2} s r}{\Delta V L} \quad \frac{N W}{L}
$$

$[W+1+2(N-1) /(N-4)]$

At any time for each user the probability of sending bit ' 1 ' is $1 / 2$, in that case Equations (21) and (22) become respectively [4]:

$$
\left\langle I_{\text {shot }}^{2}\right\rangle=e B \Re \frac{P_{s r}}{L}[2(N-1)+W]
$$

and

$$
\left\langle I_{P I I N}^{2}\right\rangle=\frac{B \Re^{2} P_{s r}^{2} N W}{2 \Delta v L^{2}}\left(W+1+\frac{2(N-1)}{N-4}\right)
$$

The thermal noise is given as [2-3]:

$$
\left\langle I_{\text {thermal }}^{2}\right\rangle=\frac{4 K_{b} T_{n} B}{R_{L}}
$$

The SNR of the FRS system can be written as

$$
S N R=\frac{I^{2}}{\left\langle i^{2}\right\rangle}=\frac{\left(I_{2}-I_{1}\right)^{2}}{\left\langle I_{\text {shot }}^{2}\right\rangle+\left\langle I_{\text {PIIN }}^{2}\right\rangle+\left\langle I_{\text {thermal }}^{2}\right\rangle}
$$

Thus, Eq. (28) based on Eq. (19), Eq. (25), Eq. (26) and Eq. (27) can be written as:

$$
S N R=\frac{\Re^{2} P_{s t}^{2} W^{2} / L^{2}}{\left(P_{s r} e B \Re / L\right)[2(N-1)+W]+\left(B \Re^{2} P_{s t}^{2} N W /\left(2 \Delta v L^{2}\right)\right)\left(W+1+\frac{2(N-1)}{N-4}\right)+4 K_{b} T_{n} B / R_{L}}
$$

To calculate the bit error rate (BER), complementary error function is employed based on SNR as in Eq. (29) [5-6, 11].

$$
P_{e}=\frac{1}{2} \operatorname{erfc}\left(\sqrt{\frac{S N R}{8}}\right)
$$

where $\operatorname{erfc}$

$$
\operatorname{erfcx}=1-\operatorname{erf} x=\frac{2}{\sqrt{\pi}} \int_{x}^{\propto} e^{-t^{2}} d t
$$

\section{RESULTS AND DISCUSSION}

Under the following assumption, the performances of the FRS scheme will be analyzed.

- The desired user is user \#1 for any case

- The interferer user is user \#2 for any case

- When transmitting in either deterministic or stochastic methods, all the users have the same power.

- The case of simultaneous transmission is considered.

In Fig. 3, the BER is plotted against the number of active users when $P_{\mathrm{sr}}=-10 \mathrm{dBm}$ at $622 \mathrm{Mbit} / \mathrm{s}$. From the figure, it is observed that the BER of FRS code is lower compared to the MQC, MFH, MDW and Hadamard codes even though the 
weight is far less, which is 4 in this case. The maximum acceptable BER of $10^{-9}$ was achieved by the FRS code with $\approx 150$ active users. This is an improvement in view of the small value of weight used and can be attributed to the fact that an FRS code has good cross correlation property that would eliminate the effects of MAI while MDW and Hadamard codes have increased the value of cross correlation as the number of users' increases. However, MQC and MFH used codes with a fixed in-phase cross correlation exactly equal to 1 for suppressing the effects of PIIN. Hence, this increases the probability of interfering which leads to performance degradation. The calculated BER for FRS was achieved for $W$ $=4$ while for MQC, MFH and Hadamard codes were for $\mathrm{W}=$ $14, W=17$, and $W=64$ respectively.

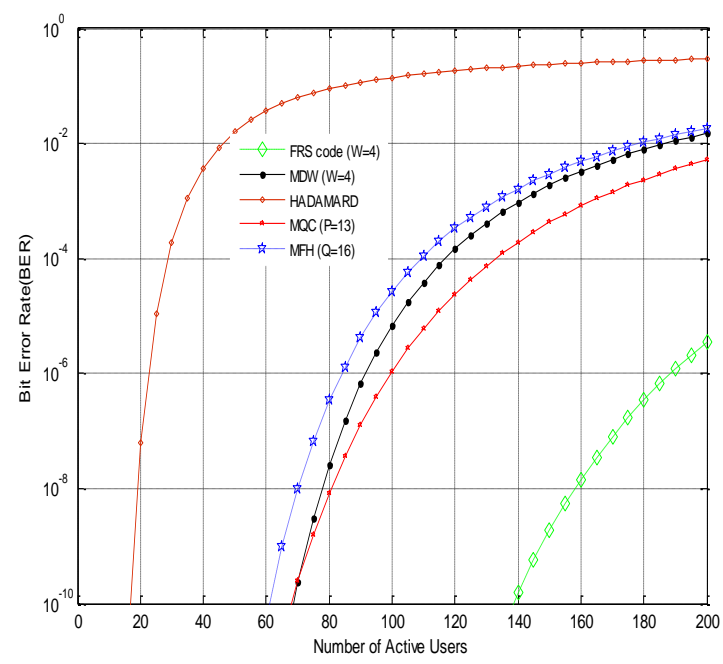

Fig. 3. BER versus number of active users when $P_{\mathrm{sr}}=-10 \mathrm{dBm}$ at $622 \mathrm{Mb} / \mathrm{s}$

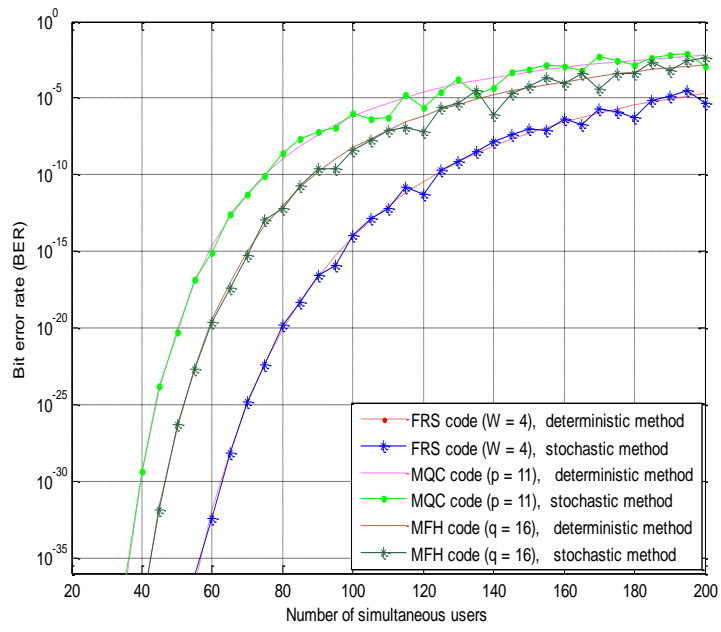

Fig. 4. BER versus number of active users when $P_{\mathrm{sr}}=-10 \mathrm{dBm}$ at $622 \mathrm{Mb} / \mathrm{s}$
In Fig. 4, the BER is sketched versus the number of active users when $P_{\mathrm{sr}}=-10 \mathrm{dBm}$ at $622 \mathrm{Mb} / \mathrm{s}$ using deterministic and stochastic methods to distribute the weight in the end to end transmission. From the figure, it can be seen that the BER of FRS code is much lower compared to the MQC and MFH codes. An acceptable BER of free error transmission was achieved by the FRS for almost two times number of users with respect to MQC and MFH codes respectively. This is because the FRS code has maximum cross correlation is one that decreasing by the factor $N-4$ to reduce the power of interference from other users. It should be pointed out that although some distortions occurred to the signals using stochastic methods, but still maintain strong signals that would recover the original information.

Fig. 5 illustrates the BER versus the effective power $P_{\mathrm{sr}}$ for 30 users at $622 \mathrm{Mb} / \mathrm{s}$ data rate considering the effects of the intensity noise, thermal noise and shot noise for FRS, MQC and MFH codes. FRS, MQC and MFH codes are adopted with the parameters $W=4,10$, and 12 respectively. The figure demonstrates that the effective power of an acceptable BER of error free transmission for the FRS code is lower than that for the MQC and MFH codes for the same number of users. As a mean of comparison, the FRS reached a BER of $10^{-9}$ at received power $-22 \mathrm{dBm}$, while $\mathrm{MQC}$ and $\mathrm{MFH}$ reached $10^{-9}$ of BER at -14 and $-12 \mathrm{dBm}$ received power. This is because the interference from other users is maximum one and reduces by the factor $\mathrm{N}-4$ for the FRS code, while for MFH and MQC codes are one as the number of simultaneous users increases.

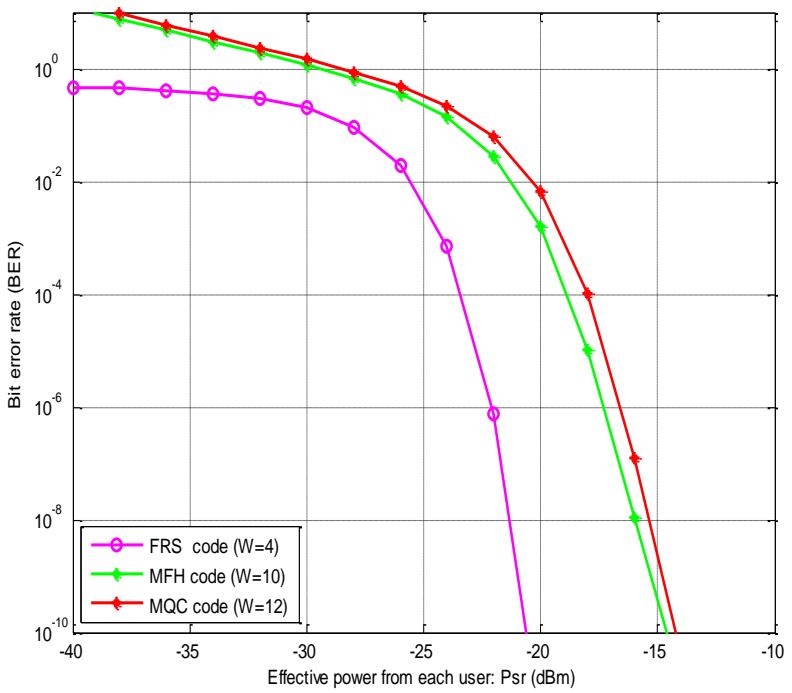

Fig. 5. $P_{\mathrm{sr}}$ versus BER when number of active users $N=30$ at $622 \mathrm{Mb} / \mathrm{s}$ 


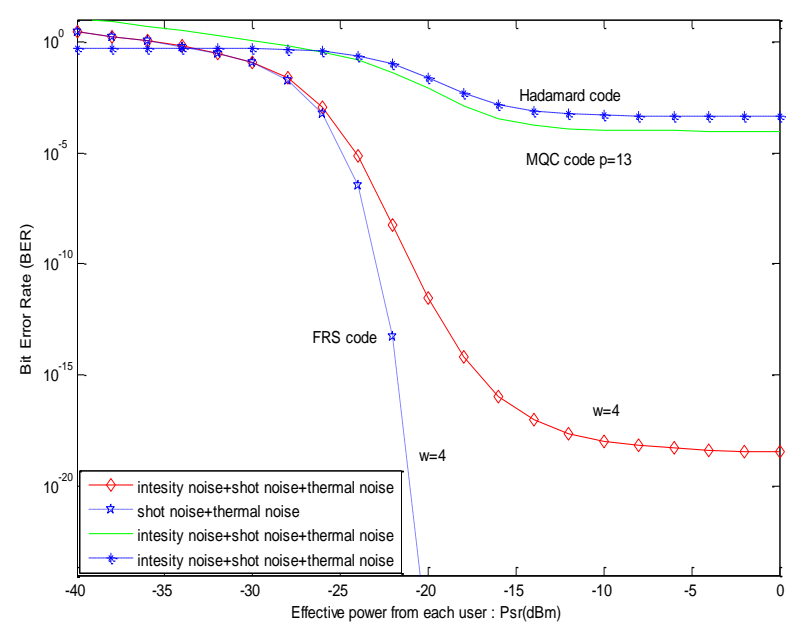

Fig. 6. BER versus effective power for FRS, MQC and Hadamard codes when number of active users are 30

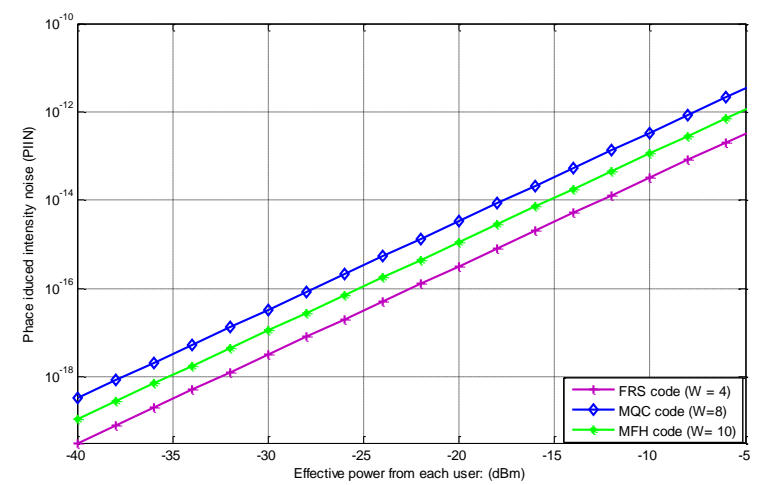

Fig. 7. PIIN versus $P_{\mathrm{sr}}$ for FRS, MQC, and MFH codes for the same number of users $(N=20)$

Fig. 6 shows the BER plotted against $P_{\text {sr }}$ when the number of active users is 30 and the data rate is $622 \mathrm{Mbit} / \mathrm{s}$. The blue line with cross represents Hadamard code while the green line symbolizes MQC for $p=13$ taking into account the effects of intensity noise, thermal noise and shot noise. The blue dashed line with stars and solid red with diamond lines represent the FRS code when only intensity noise and thermal noise, and all noises are considered respectively. From this figure, it is observed that, FRS outperforms the MQC code when all noises are considered; this is because FRS code has the ability to suppress the contribution of MAI in contrast to MQC which its MAI impact increases when a high number of users is involved.

In OCDMA systems, PIIN is interrelated to MAI due to the overlapping of spectra from different users. Fig. 7 shows the PIIN plotted against the received power for the FRS, MQC and MFH using the parameters: $\mathrm{W}=4,8$ and 10 for FRS, MQC and MFH respectively at data rate $10 \mathrm{~Gb} / \mathrm{s}$ for same number of users $(N=20)$. From the figure, it can be observed that when the received power increases, the PIIN noise for all the codes increases linearly. As shown in Fig. 7, the PIIN noise can be effectively suppressed by using the FRS code families because of $\mathrm{N}-4$ uncorrelated users even though the weight is far less than other codes (MQC, and MFH codes).

\section{CONCLUSIONS}

In this paper, a new algorithm to build a code family with $\lambda c \leq 1$ is proposed. The advantage of the FRS code family can be summarized as follows: 1) variety of code sets; 2) any positive integer number of weights can be used; 3) high scalability; 4) practical code length; 5) and easy to implement encoder/decoder structure. The properties of this code based on numerical and simulation results have been proved and discussed. It is reported that, the performance can be improved considerably when FRS code is used instead of MQC and MFH codes. It concludes that, an end-to-end transmission using deterministic, stochastic methods are having slightly difference in terms of performance, and some distortion can be noticed in stochastic method. It has been shown that the FRS code families can deter intensity noise efficiently and enhance the system performance considerably. At a big value of receiving power, the intensity noise is the major cause of signal distortion. At a low value of receiving power, thermal and shot noise sources become the major sources of limitations and the impact of thermal noise is much influential than that of shot noise. This code family has a potential to be stronger candidate for future LAN environment.

\section{ACKNOWLEDGEMENT}

This project was supported by the Deanship of Scientific Research at Sattam bin Abdulaziz University under the research project \# 2014/1/877.

\section{REFERENCES}

[1] Frigo N, Iannone P, Reichmann K. Spectral slicing in WDM passive optical networks for local access, in IEEE Proc. Europ. Conf. Optical Commun. Tech. Dig, vol. 1, pp. 119-120, 1998.

[2] Froberg ., Henion, Rao H, Hazzard B, Parikh S, Romkey, Kuznetsov M, "The NGI ONRAMP test bed: Reconfigurable WDMtechnology for next generation regional access networks," J. LightwaveTechnol, vol.18, pp. 1697-1708, 2000.

[3] Salehi J. A, Brackett C. A, "Code division multiple access techniques in optical fiber network-Part II: System performance analysis," IEEE Transaction on Communications, vol. 37, pp. 834-842, 1989.

[4] Zou Wei, Shalaby H. M. H, Ghafouri-Shiraz H, "Modified Quadratic Congruence codes for Fiber Bragg-Grating-Based SAC-OCDMA," Journal of Lightwave Technology, vol. 19, pp. 1274-1281, 2001.

[5] Wei Z, Ghafouri-Shiraz H, "Code for spectral amplitude-coding optical CDMA systems," J. Lightwave Technol, vol. 20, pp. 1284-1291, 2002.

[6] Abd T. H, Aljunid S. A, Fadhil H. A, Ahmad R. B, Junita M. N, "Enhancement of performance of a hybrid SAC-OCDMA system using dynamic cyclic shift code,” Ukr. J. Phys. Opt, vol. 13, pp. 12-27, 2012.

[7] Soma Kumawat and M. Ravi Kumar, "Generalized optical code construction for enhanced and Modified Double Weight like codes without mapping for SAC-OCDMA systems", Optical Fiber Technology, vol.30, pp.72-80, 2016.

[8] C. B. M. Rashidi, S. A. Aljunid, F. Ghania, Hilal A. Fadhila, M.S. Anuara, "New Design of Flexible Cross Correlation (FCC) Code for SACOCDMA", Procedia Engineering, vol. 53, pp. 420-427, 2013.

[9] Hassan Yousif Ahmed and K. S. Nisar, Diagonal Eigenvalue Unity (DEU) code for spectral amplitude coding-optical code division multiple access, Optical Fiber Technology. 19 (2013) 335-347.

[10] Hassan Yousif Ahmed and K.S.Nisar, Reduction of the fiber dispersion effects on MAI for long span high-speed OCDMA networks using Diagonal Eigenvalue Unity (DEU) code, Optik 124 (2013) 5765- 5773.

[11] J.W. Goodman, statistical optics, Wiley, New York, 1985. 